\title{
S2k Guidlines for Diverticular Disease and Diverticulitis: Diagnosis, Classification, and Therapy for the Radiologist
}

\section{S2k-Leitlinie Divertikelkrankheit und Divertikulitis: Diagnostik, Klassifikation und Therapie für die Radiologie}

Authors

Affiliations

\author{
A. G. Schreyer ${ }^{1}$, G. Layer $^{2}$
}

Institute of Radiology, University Hospital Regensburg, Germany

Central Institute for Diagnostic and Interventional Radiology, Ludwigshafen Hospital, Germany
Key words

abdomen

- CT

- MR imaging

inflammation

received 26.1.2015

accepted 15.3.2015

\section{Bibliography}

DOI http://dx.doi.org/

10.1055/s-0034-1399526

Published online: 27.5.2015

Fortschr Röntgenstr 2015; 187:

676-684 ๑ Georg Thieme

Verlag KG Stuttgart · New York . ISSN 1438-9029

\section{Correspondence}

\section{Prof. Andreas G. Schreyer}

Institut für Röntgendiagnostik, Universitätsklinikum

Regensburg

Franz-Josef-Strauß-Allee 11

93051 Regensburg

Germany

Tel.: ++ 49/941/9447401

Fax: ++ 49/941/9447402

andreas.schreyer@klinik.uni-

regensburg.de

\section{Abstract \\ $\nabla$}

Diverticular disease and diverticulitis represent an increasingly common disease especially in patients with advanced age. The German Society of Digestive and Metabolic Diseases (DGVS) as well as the German Society of General and Visceral Surgery (DGAV) in collaboration with the German Radiology Society (DRG) created and published S2k guidelines regarding this topic. Knowledge of the diagnosis and therapy of this common disease is extremely important for the radiologist for the daily clinical routine. In this article we review and discuss the most important clinical situations and algorithms of this disease focusing on radiological topics. Additionally, we introduce the new CCD (classification of diverticular disease) system regarding radiology.

Key Points:

- For the diagnosis of a diverticular disease a sectional imaging method should be performed. First choice should be a „qualified ultrasound examination“ followed by CT in uncertain situations or complicated disease.

- Disease classification should be done according the new CCD (Classification of diverticular disease) algorithm.

- Based on this new CCD patients can be stratified into outpatient, in-house patient and surgical treatment therapy.

Citation Format:

- Schreyer AG, Layer G. S2k Guidlines for Diverticular Disease and Diverticulitis: Diagnosis, Classification, and Therapy for the Radiologist. Fortschr Röntgenstr 2015; 187: 676-684

\section{Zusammenfassung \\ $\nabla$}

Die Divertikulose und die Divertikelkrankheit ist mit zunehmendem Patientenalter eine der häufigsten Erkrankungen des gastrointestinalen Traktes. Die neue S2k-Leitlinie zur „Divertikelkrankheit/ Divertikulitis“ der Deutschen Gesellschaft für Verdauungs- und Stoffwechselkrankheiten (DGVS) und der Deutschen Gesellschaft für Allgemein- und Viszeralchirurgie (DGAV) unter Beteiligung der Deutschen Röntgengesellschaft (DRG) wurde dazu aktuell erstellt und veröffentlicht. Dieses häufige Krankheitsbild betrifft besonders den Radiologen hinsichtlich der Diagnose- und Therapieentscheidungen in der täglichen klinischen Routine in zunehmendem Maße. Im vorliegenden Artikel werden die wichtigsten diagnostischen und therapeutischen Szenarien und Algorithmen der Erkrankung mit dem Fokus auf die Radiologie zusammengefasst und diskutiert. Zusätzlich wird die neu erstellte Klassifikation CDD (Classification of Diverticular Disease) erläutert und ihre Auswirkung auf die Radiologie besprochen.

\section{Introduction}

Diverticulosis and diverticular disease are very common changes or diseases of the gastrointestinal system in Western countries. The prevalence of diverticulosis, i.e., the presence of false diverticula without relevant clinical symptoms, is between $28 \%$ and $45 \%$, and a prevalence of over $60 \%$ can be assumed among patients over 70 years old [1, 2]. Diverticular disease is diverticulosis with clinical symptoms. It is notable that an increase in the incidence of more than $50 \%$ has been observed in the last 7 years particularly in younger patients. The most common complication of diverticular disease is diverticulitis that represents an inflammatory change of 
the intestinal wall around the diverticula and can result in covered or open perforations with consecutive stenoses, fistulas, and abscesses. The pronounced shear forces along the edge of the diverticulum can result in bleeding from the vasa recta. Despite the high prevalence and incidence of the disease, a sufficient overview of the literature allowing evidence-based diagnosis and treatment has not been provided in recent decades. The recommendations that have been practiced and taught for decades, such as the administration of antibiotics in the case of uncomplicated diverticulitis or the consideration of a second attack as an indication for surgery, were not substantiated by the literature. Therefore, there is an urgent need for new, updated, and evidence-based guidelines for the diagnosis and treatment of this common disease. The classifications of diverticulitis used to date, primarily according to Hinchey [3] in the Anglo-American region and according to Hansen and Stock [4] in Germany, are also not based on the latest scientific knowledge regarding diagnosis and course.

The significance of imaging primarily via ultrasound and computed tomography in the clinical routine must be reevaluated in the context of therapeutic consequences. This raises the question as to the extent to which smaller covered perforations or small abscesses affect the further course of treatment.

\section{Method}

\section{$\nabla$}

Based on these questions and consideration, the German Society of Digestive and Metabolic Diseases (DGVS) together with the German Society of General and Visceral Surgery (DGAV) created S2 guidelines regarding diverticular disease and diverticulitis. In medical guidelines according to the system of the AWMF (Association of the Scientific Medical Societies in Germany), a differentiation is made between S1, S2, and S3 based on the quality level of the development method. S1 guidelines represent only an informal consensus of an expert group. In the case of the S2 classification, a subclassification into S2k and S2e guidelines has been used since 2004. In the case of S2k (consensus-based) guidelines, representatives of every relevant target audience should be included in the guideline development group. Formal consensus development is required. A systematic literature search must be performed for S2e (evidence) guidelines. In $\mathrm{S} 2 \mathrm{k}$ guidelines every recommendation in the framework of the structured consensus development process should be discussed with neutral moderation and then evaluated based on the strength of the consensus. In contrast to S2e guidelines, the recommendations from S2k guidelines do not receive evidence and recommendation grades since a systematic review of the evidence in the literature is not performed [5]. At the highest quality level of S3 guidelines, all elements of the consensus development process must be present. The strength of the consensus ( $\odot$ Table 1 ) and the nomenclature of the strength of the recommendation ( 0 Table 2 ) corresponded to the usual recommendations for S2k guidelines in the present methods. A systematic literature search beginning with 9/1/1998 and ending with the day of the guidelines conference (3/16/ 2013) was performed for the guidelines. The Danish guidelines published in 2012 were also taken into consideration
Table 1 Definition of the strength of consensus for votes regarding the $52 \mathrm{k}$ guidelines.

\begin{tabular}{|ll|}
\hline strong consensus & agreement $>95 \%$ of participants \\
\hline consensus & agreement $>75-95 \%$ of participants \\
\hline majority agreement & agreement $>50-75 \%$ of participants \\
\hline no consensus & agreement $<50 \%$ of participants \\
\hline
\end{tabular}

\begin{tabular}{|ll}
\hline Table 2 & Nomenclature of strength of recommendation based on votes. \\
\hline formulation & strength of the recommendation \\
\hline "to be implemented" & strong recommendation \\
\hline "should be implemented" & recommendation \\
\hline "can be implemented" & open recommendation \\
\hline "should not be implemented" & negative recommendation \\
\hline "not to be implemented" & strong negative recommendation \\
\hline
\end{tabular}

[6]. In total 6 work groups of experts were established (group 1: anatomy, pathogenesis; group 2: clinical appearance, natural course, complications, epidemiology; group 3: diagnosis and staging; group 4: conservative treatment, medications, nutrition, lifestyle; group 5: indications: outpatient/inpatient treatment, conservative/surgical treatment; group 6: surgical procedure). The German Society of Ultrasound in Medicine (DEGUM) and the German Radiology Society (DRG) were represented by two delegates. A radiology representative was assigned to work group 3 (diagnosis and staging) and to work group 5 (indication: outpatient/inpatient treatment, conservative/surgical treatment). After initiation of the guidelines in January 2012, they were concluded in March 2013 at the guidelines conference and were then published in February 2014 [7].

\section{Definitions \\ $\nabla$}

Colonic diverticula are defined as acquired outpouchings of the mucosa and submucosa through weak spots in the colon wall. By definition, these are not true diverticula but rather false diverticula. The outpouching occurs in portions of the submucosa through loci minoris resistentiae in the immediate vicinity of intramural blood vessels represented by the vasa recta. It is assumed that false diverticula therefore primarily occur in the sigmoid colon because numerous vasa recta are located in this intestinal region and high intraluminal pressures are present. In the case of local inflammation that typically arises from colonic diverticula (peridiverticulitis), the inflammation often spreads to the entire intestinal wall (focal pericolitis). This explains further serious complications such as abscess or fistula formation and covered or open perforation with peritonitis and stenosis. In addition, the proximity of the vasa recta can often result in diverticular bleeding in this region. The susceptibility of colonic diverticula to localized inflammation is explained by the fact that the vasa recta are locally compressed with consecutive hypoperfusion, thus predisposing the areas to inflammatory changes [8]. Moreover, bacteria-laden stool with additional mechanical irritations can be assumed in this intestinal segment. Covered perforations are the result of local inflammation and are the origin of local abscess and fistula formation. Open perforations can also occur without in- 
flammatory changes and are primarily due to weakening at the tip of the diverticulum [9]. The blood vessels under pressure at the necks of the diverticula and at the tips of the diverticula are susceptible to erosion and can result in significant sigmoid bleeding even without a concomitant inflammatory change.

The term "diverticular disease of the large intestine" should be used when diverticulosis results in symptoms and/or complications. A persistent or recurrent disease course without diverticulitis being visible on imaging is considered uncomplicated diverticular disease. The term "acute diverticulitis" should be used in the case of inflammation of false diverticula and neighboring structures. "Complicated diverticulitis" is present when complications such as perforation, fistulas, or abscesses have occurred. "Chronic diverticulitis" is defined by recurrent or persistent inflammatory episodes that can result in further complications such as stenoses or fistulas over the course of the disease.

\section{Epidemiology and lifestyle \\ $\nabla$}

With respect to age and gender distribution and genetic predisposition, there is a clear connection between the prevalence of diverticula or diverticular disease and advanced age. In addition to environmental factors, genetic predispositions play an important role in the development of the disease. The prevalence for diverticulosis is approximately $13 \%$ for the Western population under 50 years old, approximately $30 \%$ for 50 to 70 -year-olds, and approximately $50 \%$ for the group between 70 and 85 years old. Up to $66 \%$ of people over the age of 85 have diverticulosis [ 10 , 11]. Diverticular disease is also age-dependent. According to the latest studies, primarily the number of younger patients with diverticular disease is currently on the rise. The incidence increased most significantly between 1998 and 2005 primarily in the group of 18 to 44-year-olds from $151 / 1$ million to $251 / 1$ million followed by the group of 45 to 64 -year-olds (659/1 million to $777 / 1$ million) with stable or decreased frequency among patients of an advanced age [12]. There is no clear data regarding the gender distribution of patients with diverticulosis [10]. In the case of a genetic influence on the development of diverticular disease, the proportion of genetic factors is estimated at $40 \%$ compared to $60 \%$ based on environmental factors [13].

With respect to the influence of eating habits or lack of dietary fiber, older studies from the 1960 s examined a geographic and thus diet-based difference between diverticulosis and diverticular disease [14]. It was thus postulated that the traditional high-fiber diet in Africa and Asia results in a lower probability of diverticulosis and diverticular disease, while Afro-American and Japanese immigrants have an increased prevalence of diverticulosis after adaptation to Western dietary habits. Overall, providing proper scientific proof of a connection between a fiber-rich diet and diverticulosis is extremely difficult. Two case control studies $[15,16]$ and two cross-sectional studies did not find a relevant effect of fiber consumption on the occurrence of diverticulosis [17, 18]. The decades-long hypothesis that increased consumption of nuts and grains resulted in an increased rate of complications in diverticular disease due to the nuts becoming trapped in the necks of the diverticula also could not be con- firmed. In contrast, it was able to be shown that increased consumption of nuts and grains tended to reduce the occurrence of diverticular disease [19]. The consumption of red meat also could not be convincingly proven as an influencing factor for the occurrence of diverticulosis [17]. The regular consumption of alcohol, in particular high-proof alcohol, probably correlates with an increased occurrence of diverticular disease and complications. However, a relevant correlation with coffee consumption and smoking could not be proven on the basis of currently available inhomogeneous studies. Also in the case of obesity, the current data regarding increased incidence of diverticulosis is inhomogeneous and questionable. Therefore, no relevant connection between BMI and diverticulosis could be shown in a prospective cohort study and a cross-sectional study $[18,20]$.

In the literature the mortality rate of acute cases of diverticulitis treated on an inpatient basis is between $0 \%$ and $13 \%$, while it is significantly higher at $8-24 \%$ in cases of complicated diverticulitis and in patients under immunosuppresive therapy [21]. In principle, the mortality rate is higher in the first year after a complicated case of diverticulitis with a 2.5-fold increase after fistula formation and even a 4.5-fold increase after perforation being described. A mortality rate of $2.25 \%$ in the case of diverticular bleeding is specified in retrospective series [22]. Even though diverticulosis and colorectal tumors have many commonalities with respect to their epidemiology primarily regarding lifestyle and age, there is no increased prevalence of colorectal carcinomas in patients with diverticulosis.

In summary, a lower intake of fiber probably increases the risk of diverticular disease but the studies are not clear with respect to the occurrence of diverticula. Even in the case of obesity, only an increased risk of diverticular disease can be assumed but increased physical activity tends to lower the risk of diverticular disease.

\section{Diagnosis \\ $\nabla$}

For further treatment and diagnosis, exact imaging with differential diagnosis in nonspecific abdominal symptoms of the disease is necessary. In addition to palpation, percussion, and auscultation of the abdomen, a rectal examination, a temperature measurement, and determination of the inflammatory parameters in the blood and a urinalysis must be performed. Typical symptoms of diverticular disease are pain in the left lower abdomen associated with increased inflammation values. CRP is the best established and validated lab value in acute diagnosis and for followup. CRP values tend to correlate with complicated disease courses. A CRP value of greater than $5 \mathrm{mg} / 100 \mathrm{ml}$ is typical for diverticulitis, while CRP values of greater than $20 \mathrm{mg} /$ $100 \mathrm{ml}$ with a positive predictive value of $69 \%$ indicate perforation. There is a negative predictive value (NPV) for perforation of $79 \%$ in CRP values of less than $5 \mathrm{~m} / 100 \mathrm{ml}$ [23].

\section{Imaging}

The new guidelines require cross-sectional imaging to verify diagnosis of diverticulitis. This recommendation is based on the fact that a lack of imaging results in an increased di- 
agnostic error rate. Therefore, clinical diagnosis without imaging reaches sensitivities of only between $64 \%$ and $71 \%$ [24, 25].

"Qualified abdominal ultrasound" should be used as the primary cross-sectional imaging procedure for the initial and follow-up diagnostic workup of acute diverticulitis. An acoustic frequency $\geq 3.5 \mathrm{MHz}$ using recommended high-resolution ultrasound frequencies $>5 \mathrm{MHz}$ while applying controlled compression at the site of maximum pain is recommended for ultrasound. When performed by experienced examiners in a targeted manner, ultrasound reaches a sensitivity and specificity of $98 \%$ [26]. Comparative studies between ultrasound and CT are available as older prospective studies that show an accuracy of $84 \%$ and a sensitivity of $85 \%$ for ultrasound and $91 \%$ for CT [27]. The literature shows that ultrasound is ideally suited for uncomplicated acute diverticulitis in particular. It must be noted that pathologies with a difficult anatomical location, such as distant mesenteric abscesses or deep-seated cases of diverticulitis of the distal sigmoid colon, often cannot be properly visualized with ultrasound [28]. In the case of such locations, CT should be given preference over ultrasound alone particularly in the evaluation of the local situation in the case of an indication for emergency surgery.

Metaanalyses show that ultrasound and CT have comparable values in the evaluation of diverticular disease [29, 30]. However, it is interesting here that the good results for ultrasound in the metaanalyses were primarily due to the fact that the sonographic studies had a higher study quality compared to CT and MRI studies [31].

Based on the data from the literature and the consensus, the guidelines therefore require ultrasound and/or computed tomography to be used as the diagnostic method in the case of suspicion of diverticulitis. A colonic contrast enema should no longer be performed since this examination does not provide further information and involves high radiation exposure.

Inflamed diverticula can be directly detected on both CT and ultrasound on the basis of bowel wall thickening of more than $3 \mathrm{~mm}$ with increased contrast enhancement on CT, MRI, and CEUS as a direct sign. Perifocal mesenteric injection and free abdominal fluid as a nonspecific result of inflammation are indirect signs seen with cross-sectional imaging. Covered or free perforations must be detected by direct detection of air inclusions outside the intestinal lumen.

\section{Computed tomography \\ $\nabla$}

Excellent sensitivities and specificities of CT for diagnosis in the case of suspicion of acute diverticulitis have already been proven in older studies with single-row device configurations. For this reason there are no comparative studies with the new multi-row devices which certainly further improve diagnostic accuracy but do not provide a relevant clinical benefit given a sensitivity of already almost $100 \%$. There are not enough high-quality comparative studies regarding the necessity of intravenous contrast enhancement, the use of rectal contrast enhancement, and the selection of an oral contrast agent. The requirement described here for $\mathrm{CT}$ in diverticular disease therefore represents a description of the current actual status based on the current literature and our own experience. There is a need for radiologists to further optimize radiological protocols for CT examination. In most cases, contrast-enhanced examination with typically positive oral and rectal contrast enhancement is performed in German-speaking countries. If necessary, positive contrast enhancement is to be given preference since it allows possible abscesses to be better differentiated from the intestinal lumen or contrast agent escape can be directly detected. Examination is typically performed in the portal venous contrast phase at a tube voltage of between 100 and $120 \mathrm{kVp}$ and a tube current of approximately $120 \mathrm{mAs}$. As already mentioned, this description of the "actual" status must be critically examined in the future. Therefore, the use of a low-dose technique is possible as already shown in studies with a reduction of up to $30 \mathrm{mAs}$ with similar results to those of conventional CT at a dose of only $3 \mathrm{mSv}$ [32]. Recently introduced techniques such as iterative reconstructions and innovative tube and detector techniques allow a further reduction of radiation exposure. It must be determined whether the customarily used oral and rectal contrast enhancement is really necessary in every case. It was able to be shown in individual studies that diverticular disease detection rates that are similar to those obtained with contrast enhancement can be achieved even without rectal and oral contrast enhancement [32]. Large systematic radiological studies are also urgently needed here.

The guidelines are still relatively conservative regarding the use of MRI for diagnosis based on the current literature. To date, this method has only been evaluated in small and selected patient collectives, primarily in Germany [33-35]. Due to a lack of data and availability, MRI is not recommended for routine diagnosis of diverticulitis. Radiologists urgently need to clinically evaluate the status of MRI for the diagnosis of diverticular disease using the current equipment generation. Developments ranging from newly introduced high-resolution T2-weighted $3 \mathrm{D}$ sequences to the quick implementation of diffusion-weighted sequences to evaluate inflammation without contrast agent have significant unrealized potential that must be further developed. The main criticism of MRI, i.e. that small quantities of free air may not be able to be detected, must be critically examined with respect to clinical classification and treatment since small quantities of free air in covered perforations do not require immediate surgical intervention. Therefore, MRI of the pelvis is a very interesting modality for use as an alternative radiation-free method in the medium term.

\section{Endoscopic diagnosis and therapy}

$\nabla$

The conventional wisdom that colonoscopy should never be performed in the case of acute diverticulitis was challenged based on new evidence and studies. In principle, it is stated that endoscopic methods should not be performed. However, it has now been postulated that in the case of select indications, such as an uncharacteristic clinical image or disease course, colonoscopy can be performed with an at most slightly increased risk of perforation in acute diverticulitis if a covered perforation or abscess has been ruled out on computed tomography. This statement is based among other things on a study in 54 patients with diverticulitis in 
whom colonoscopy resulted in a perforation in $1.9 \%$ of cases. However, in the subgroup of 39 of 54 patients in whom a covered perforation or abscess had been ruled out by $\mathrm{CT}$, not a single perforation was observed [36]. In the specified study, colonoscopy allowed real-time diagnosis of two CT-negative adenocarcinomas among other things.

The diagnosis or differentiation of a carcinoma of the sigmoid colon or sigmoid diverticulitis is not reliably possible even using the latest cross-sectional imaging methods [37, 38]. Therefore, the indication for colonoscopy after conservative treatment of diverticulitis remains liberal and has not been changed by the guidelines, and colonoscopy to rule out other relevant pathologies is required prior to elective resection of the sigmoid colon ( $\bullet$ Fig. 1 ).

Regarding imaging, reference is made again to the modified diagnostic algorithm from the guidelines in the case of suspicion of sigmoid diverticulitis with consideration of the patient history, the clinical findings, and the basic lab work ( 0 Fig. 1 ). In the case of a clinically acute abdomen, ultrasound is followed by further radiological evaluation, routinely performed by $\mathrm{CT}$, which usually provides risk stratification for decisions regarding further surgical and conservative therapeutic measures. The potential of CT for differential diagnosis should be given particular emphasis here. In the case of the clinical absence of an acute abdomen, the decision is primarily based on the lab parameter CRP. In the case of a normal CRP value, diverticulitis is ruled out solely by ultrasound examination. Colonscopy is subsequently performed in the interval. In the case of a significantly elevated CRP value, the probability of a perforation and abscesses is very high so that contrast-enhanced abdominal CT should be performed in the case of unclear ultrasound findings to determine the further therapeutic course and to rule out penetration and abscesses.

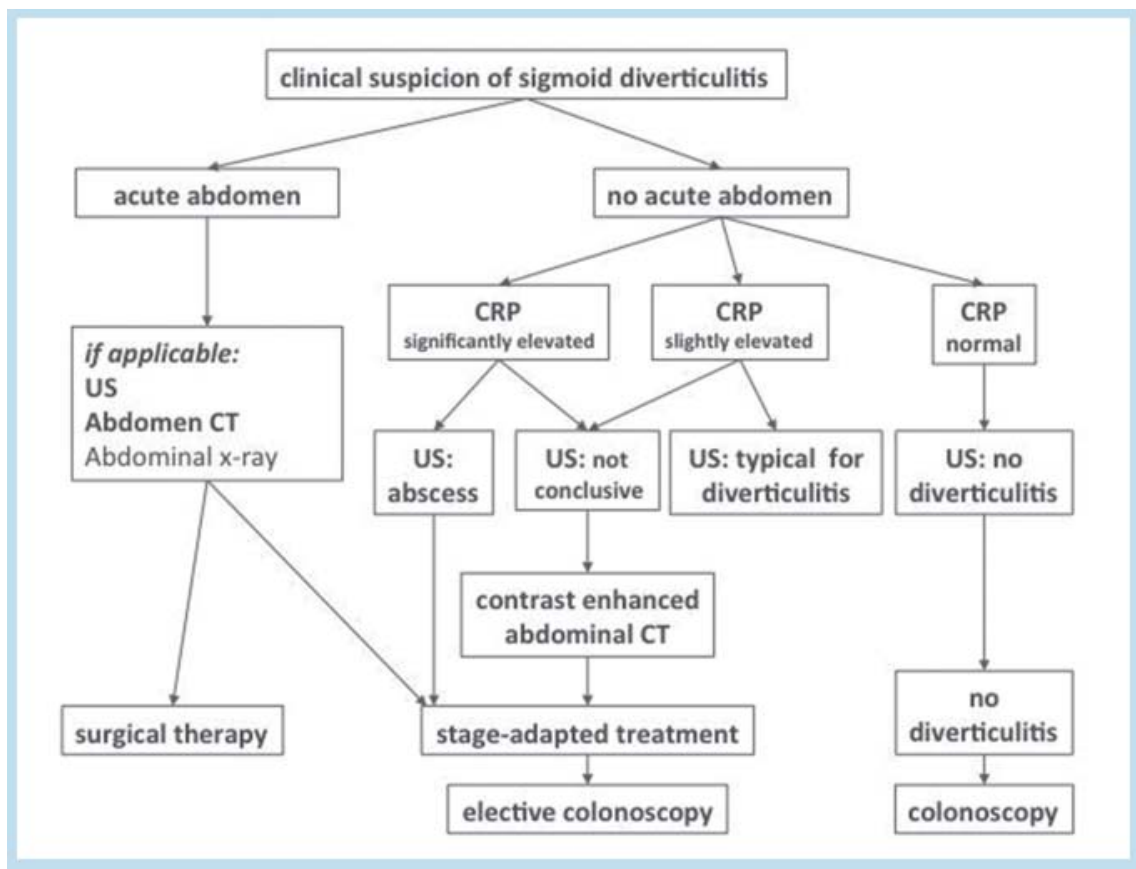

\section{Classification}

\section{$\nabla$}

To date, two classifications for diverticular disease have been mainly used in the literature. The Hinchey classification that is primarily known and prevalent in Anglo-American countries stratifies surgical procedures on the basis of varying degrees of macroscopically perforated diverticulitis with abscesses and free perforation [3]. This classification does not sufficiently address chronic changes. The focus of this classification is also not on the categorization of the disease without further surgical implications. The classification of Hansen and Stock primarily includes the different forms of diverticulitis with important categories for outpatient treatment [4]. Microperforations and macroperforations and abscesses of varying degrees and location are not taken into further consideration in the classification. After in-depth discussion by the guideline participants and an additional online discussion with renewed Delphi consensus after conclusion of the guidelines conference, an interdisciplinary attempt was made to create a new classification of diverticular diseases (CDD) ( $\bullet$ Table 3 ).

The new classification has the following primary categories: Type 0 does not have pathological significance and represents an incidental finding. Type 1 represents uncomplicated diverticular disease or diverticulitis without perforation. In the case of type 1 diverticular disease is present but at most a phlegmonous reaction of the surrounding tissue with indirect signs on imaging can be detected ( $\bullet$ Fig. 2 ). Type 2 represents complicated diverticulitis and is subdivided into type $2 \mathrm{a}-$ microabscess $(<1 \mathrm{~cm})$ ( $\bullet$ Fig. 3 ), type $2 \mathrm{~b}-$ macroabscess ( $>1 \mathrm{~cm}$ with paracolic and mesocolic abscesses) ( $\bullet$ Fig. 4 ), and Type $2 c-$ free perforation ( $\bullet$ Fig. 5 ). The chronic forms are differentiated in type 3 to provide therapy-relevant categorization. Type 4 refers to diverticular bleeding, especially because diverticular bleeding is not necessarily a result of diverticulitis but rather can develop on the basis of diverticulosis.
Fig. 1 Modified diagnostic algorithm in the case of clinical suspicion of sigmoid diverticulitis from the $\mathrm{S} 2 \mathrm{k}$ guidelines regarding diverticular disease/diverticulitis. In the case of a lack of the clinical picture of an "acute abdomen", the CRP value should be used as the gatekeeper for further diagnosis and treatment. High-resolution B-mode ultrasound is typically performed as the basic examination. In the case of unclear findings or clinically urgent suspicion of complicated diverticular disease, abdominal CT should be performed as quickly as possible for differential diagnosis. 
As in the previous classification systems, there is no evidence in clinical studies regarding treatment and outcome evaluation for this newly created classification that is intended to avoid the problems of the two largest classification systems currently in use. A first evaluation of this classification is currently being performed by several participating clinics of the guidelines conference. Its use in practice must still be proven. However, this classification is clearer than the Hansen-Stock or Hinchey classification with respect to radiological diagnosis. Particularly the differentiation of type 1 and type 2 between uncomplicated and complicated diverticular disease can be performed reliably in most cases with radiology. Covered perforations and smaller abscesses $<1 \mathrm{~cm}$ are equivalent in type $2 \mathrm{a}$. Treatment-relevant categorizations can certainly be made in the future by radiologists based on this classification. Radiological studies are urgently needed here.

\section{Treatment}

\section{$\nabla$}

Regarding further treatment, cases must be categorized as complicated or uncomplicated diverticular disease, as in the new classification. In acute uncomplicated diverticulitis without risk indicators, antibiotic therapy is not necessary according to the new guidelines. Acute uncomplicated diverticulitis (CDD type 1) should be primarily treated conservatively. Successfully treated acute uncomplicated diverticulitis is not an indication for surgery in the further disease course. Patients with complicated diverticulitis, i. e., CDD type 2 patients, should be treated on an inpatient basis according to the guidelines. Parenteral substitution should only be performed in the case of a lack of oral intake. Antibiotic therapy should be performed in all complicated cases of diverticulitis. Antibiotic therapy should include the expected polymi-

\begin{tabular}{|c|c|c|}
\hline \multicolumn{3}{|c|}{ classification of diverticular disease - CDD } \\
\hline type 0 & asymptomatic diverticulosis & \\
\hline & & $\begin{array}{l}\text { incidental finding; asymptomatic } \\
\rightarrow \text { no disease }\end{array}$ \\
\hline type 1 & acute uncomplicated diverticular disease/diverticulitis & \\
\hline type 1a & $\begin{array}{l}\text { diverticulitis/diverticular disease without reaction } \\
\text { of surrounding tissue }\end{array}$ & $\begin{array}{l}\text { symptoms related to diverticula } \\
\text { signs of inflammation (lab) } \\
\text { optional: Typical cross-sectional imaging }\end{array}$ \\
\hline type 1b & $\begin{array}{l}\text { diverticulitis with phlegmonous reaction of sur- } \\
\text { rounding tissue }\end{array}$ & $\begin{array}{l}\text { signs of inflammation (lab) } \\
\text { obligatory: Cross-sectional imaging } \rightarrow \text { Phleg- } \\
\text { monous diverticulitis }\end{array}$ \\
\hline type 2 & acute complicated diverticulitis as in $1 b$, additionally: & \\
\hline type 2a & microabscess & $\begin{array}{l}\text { covered Perforation, small abscess } \\
(\leq 1 \mathrm{~cm}) \text {; minimum paracolic air }\end{array}$ \\
\hline type 2b & macroabscess & paracolic or mesocolic abscess (> $1 \mathrm{~cm}$ ) \\
\hline type 2c & free perforation & $\begin{array}{l}\text { free perforation, free air/fluid } \\
\text { generalized peritonitis }\end{array}$ \\
\hline type $2 \mathrm{c} 1$ & purulent peritonitis & \\
\hline type 2 c2 & fecal peritonitis & \\
\hline type 3 & $\begin{array}{l}\text { chronic diverticular disease } \\
\text { recurrent or chronic symptomatic diverticular disease }\end{array}$ & \\
\hline type 3a & $\begin{array}{l}\text { symptomatic uncomplicated diverticular disease } \\
\text { (SUDD) }\end{array}$ & $\begin{array}{l}\text { typical symptoms } \\
\text { signs of inflammation (lab): optional }\end{array}$ \\
\hline type 3b & recurrent diverticulitis without complications & $\begin{array}{l}\text { signs of inflammation (lab) present } \\
\text { cross-sectional imaging: typical }\end{array}$ \\
\hline type 3c & recurrent diverticulitis with complications & detection of stenoses, fistulas, conglomerate \\
\hline type 4 & diverticular bleeding & detection of the source of the bleeding \\
\hline
\end{tabular}

Table 3 Based on the classifications of Hinchey[3] and Hansen and Stock[4], the new CDD (classification of diverticular disease) was created with a differentiation between uncomplicated (type 1), complicated (type 2), and chronic (type 3) diverticular disease. Among cases of complicated diverticular disease, a further differentiation is made depending on the therapeutic approach between microabscess, macroabscess, and free perforation.
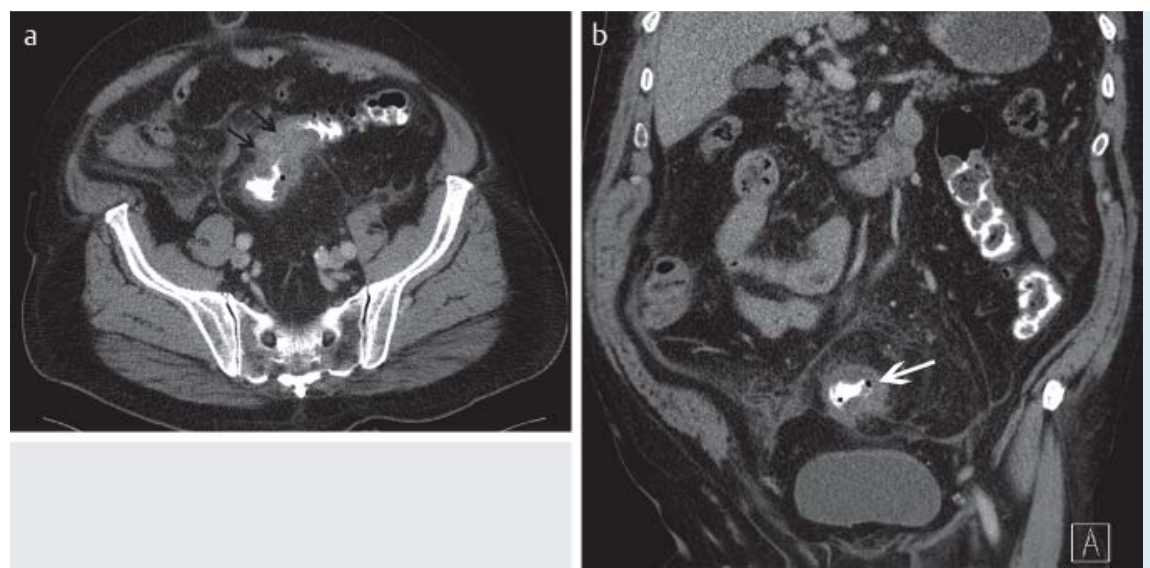

Fig. 2 Axial $\mathbf{a}$ and coronal $\mathbf{b}$ CT of the abdomen and pelvis with intravenous, oral and rectal contrast enhancement in a 74-year-old patient with lower abdominal pain: Diverticular disease type 1b according to CDD with wall thickening (arrows) and increased contrast enhancement in the sigmoid colon with phlegmonous reaction of the surrounding tissue. 
crobial spectrum of pathogens (e.g. ampicillin, sulbactam, moxifloxacin, etc.).

Surgery with delayed urgency is to be performed only in the case of a lack of response to conservative treatment of complicated diverticulitis (type 2a/b). A lack of response to adequate treatment is considered a lack of response to intravenous double antibiotic therapy (e.g. cibrofloxacin und metronidazole) with interventional abscess drainage and increasing signs of sepsis. In the case of a successfully treated case of complicated diverticulitis (macroperforation, abscess type $2 \mathrm{~b}$ ), surgery in an inflammation-free interval is

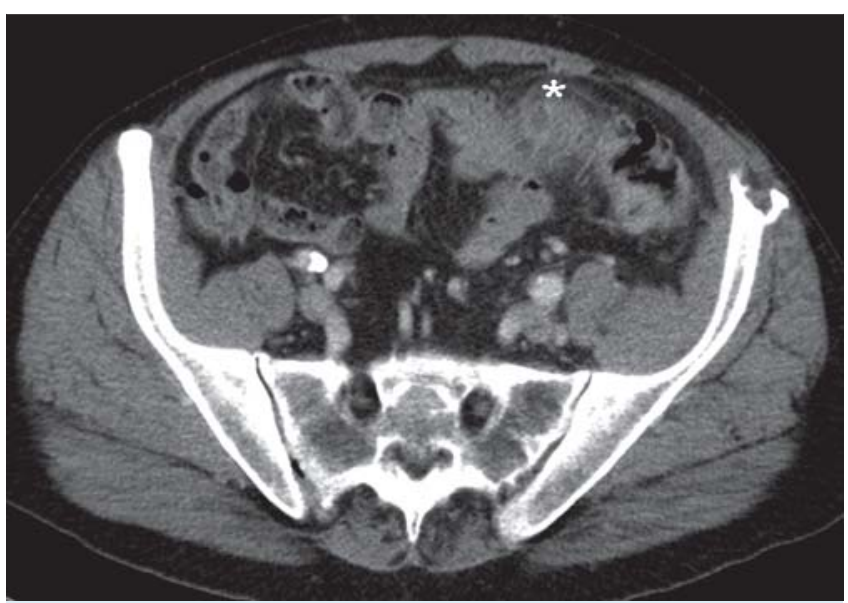

Fig. 3 Axial CT of the pelvis with intravenous contrast enhancement in a 58-year-old patient: Diverticular disease type 2a according to CDD with covered perforation and small intramural abscess $\left({ }^{*}\right)$ in the thickened inflammatory sigmoid colon. recommended. In the case of the presence of retroperitoneal or paracolic abscesses, drains can be placed by a radiologist. In the case of smaller abscesses that cannot be reliably punctured, daily inflammation monitoring and conservative therapy should be performed. According to the literature, abscesses can be detected on computed tomography in approximately $15 \%$ of patients with acute diverticulitis $[39,40]$. In the case of larger abscesses $>4 \mathrm{~cm}$, abscess drainage in combination with antibiotic therapy should be performed to prevent the need for emergency surgery. Smaller abscesses less than $3-4 \mathrm{~cm}$ can be cured in almost all cases by antibiotics alone [40]. There is a case control study in which the results for patients treated solely with antibiotic treatment in the case of abscesses that cannot be punctured were not inferior to the results of combined treatment via CT-guided drainage system plus antibiotics in the further clinical course [41]. There are no prospective randomized case collections here. However, guideline recommendations should be followed and larger abscesses $>4 \mathrm{~cm}$ should be treated with antibiotic therapy and be drained by a radiologist ( $\bullet$ Fig. 4 ) while smaller abscesses $<4 \mathrm{~cm}$ can often be sufficiently treated by antibiotic therapy alone. According to the guidelines, patients with diverticulitis-related abscesses that cannot be drained by intervention or that do not respond to conservative therapy within 3 days should be surgically drained. In the case of proof of free perforation and peritonitis, emergency surgery is recommended immediately after diagnosis.

In the case of chronic recurrent uncomplicated diverticulitis (CDD type 3b), surgery should be performed in an inflammation-free interval following individual case evaluation. There is no indication to justify elective operations based on the number of preceding episodes. The often still preva-
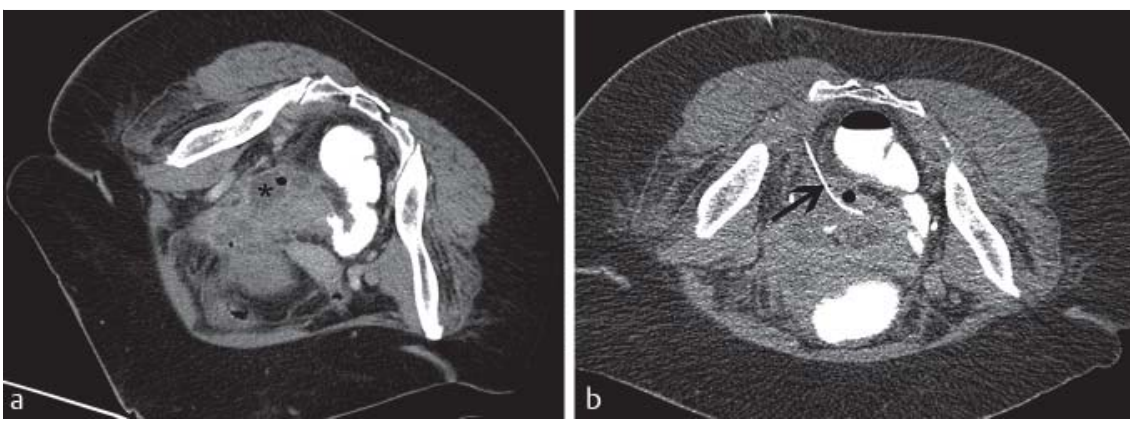

Fig. 4 Axial CT of the pelvis (patient in prone position) in a 64-year-old patient with perforated sigmoid diverticulitis with macroabscess $\left({ }^{*}\right)$ : Diverticular disease type $2 \mathrm{~b}$ according to CDD a. A $10 \mathrm{~F}$ drain (arrow) was placed in the abscess for drainage $\mathbf{b}$
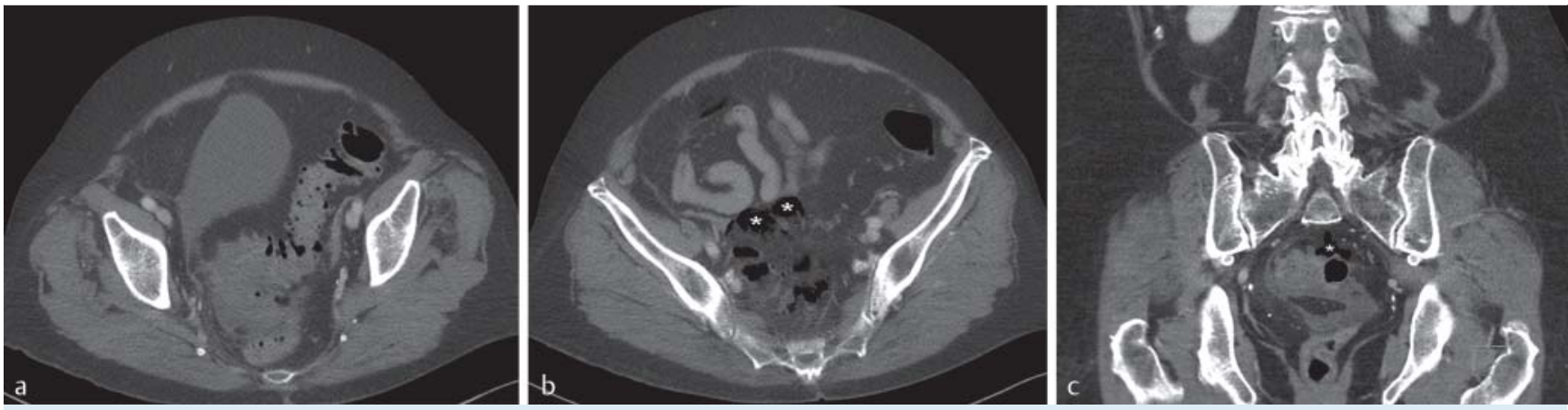

Fig. 5 Axial a, b and coronal c CT of the abdomen and pelvis with intravenous, oral and rectal contrast enhancement in an 84-year-old patient: Diverticular disease type $1 \mathrm{c}$ according to CDD with perforated sigmoid diverticulitis with free abdominal air $\left({ }^{*}\right)$. 
lent conventional wisdom that resection should be performed after a second episode is based on over 40 years of old data from a medical era without modern antibiotics [8]. With regard to the surgical procedure, the German guidelines contain the new information that laparoscopic or laparoscopically assisted operations are to be given preference over open resection if there is no contraindication. Sigmoid resection with primary continuity restoration or anastomosis with upstream ileostomy should be performed as a standard intervention.

\section{Handling complications}

$\nabla$

Painless lower gastrointestinal bleeding can be largely attributed to diverticular bleeding (35\%) and bleeding due to angiodysplasia (21\%) [42]. It must be noted that diverticular bleeding is not to be interpreted as a complication of acute inflammatory intestinal wall changes. Instead diverticulosis with consecutive shear forces of the diverticula at the entry point of the vasa recta is considered to be etiological. In principle, this type of bleeding should be endoscopically diagnosed and treated. Alternatively, in the case of bleeding that cannot be identified and treated endoscopically, the guidelines specify CT angiography for diagnosis and conventional DSA for determining the location of the diverticular bleeding. Angiography with embolization in the case of identification of the site of bleeding can be performed in individual cases. In all other cases with sustained bleeding or in the case of clinically relevant recurrent bleeding after endoscopic or angiographic initial hemostasis, surgery should be performed immediately.

Diverticular bleeding is often self-limiting (70-90\%) [22]. Patients with self-limiting diverticular bleeding or bleeding that was successfully treated interventionally should not undergo surgery.

\section{Summary and discussion}

$\nabla$

The new S2k guidelines regarding diverticular diseases address the broad range of diverticular diseases for the first time in a systematic and interdisciplinary manner. Many previously established statements that have never been proven in modern medicine were able to be dismissed in these guidelines. For example, there is currently no indication for surgical resection after a second episode, laparoscopic resection methods should be given preference, and patients should only be admitted in the case of complicated diverticular disease. The ambitious attempt to create a new classification in new guidelines based on the two most established systems is an important condition and requirement for the clinical use of these guidelines. The clear categorization as complicated and uncomplicated diverticular diseases with corresponding therapeutic implications regarding inpatient treatment, antibiotic administration, or surgery provides clearer recommendations. For radiologists, the diagnostic practice in Germany of B-mode ultrasound being the primary diagnostic method performed by trained and certified physicians and not by medical support staff as is typically the case in Anglo-American countries has been validated. However, the high quality that is often provided in the daily routine is not always to be performed in emergency care. In the case of suspicion of a complicated course or unclear findings, contrast-enhanced computed tomography should be performed as quickly as possible as the next step. Only in the case of CRP values that are not elevated can contrast-enhanced CT initially be dispensed with since the presence of complicated diverticulosis is not likely. However, especially since many clinical symptoms of diverticular disease are very nonspecific, computed tomography as a powerful radiological tool will continue to be important for further differential diagnostic workup in the case of upper and lower abdominal pain. In the near future radiologists should optimize $\mathrm{CT}$ protocols for example with low-dose techniques and examine MRI with respect to its significance in the diagnosis of diverticular disease according to the new CDD classification. In the case of larger abscesses and diverticular bleeding, competent radiologicalinterventional care must be ensured.

\section{References}

1 Golder M, Ster IC, Babu P et al. Demographic determinants of risk, colon distribution and density scores of diverticular disease. World journal of gastroenterology 2011; 17: 1009-1017

2 Hughes LE. Postmortem survey of diverticular disease of the colon. I. Diverticulosis and diverticulitis. Gut 1969; 10: 336 - 344

3 Hinchey EJ, Schaal PG, Richards GK. Treatment of perforated diverticular disease of the colon. Advances in surgery 1978; 12: 85-109

4 Hansen 0 , Stock W. Prophylaktische Operation bei der Divertikelkrankheit des Kolons - Stufenkonzept durch exakte Stadieneinteilung. Langenbeck's archives of surgery/Deutsche Gesellschaft fur Chirurgie 1999; 115: $1257-1260$

5 AWMF. Klassifikation S2-Leitlinien; 2014, http://www.awmf.org/leitlinien/awmf-regelwerk/ll-entwicklung/awmf-regelwerk-01-planung-und-organisation/po-stufenklassifikation/klassifikation-s2eund-s2k.html (accessed 19.12.2014 2014)

6 Andersen JC, Bundgaard L, Elbrond $H$ et al. Danish national guidelines for treatment of diverticular disease. Danish medical journal 2012; 59: $\mathrm{C} 4453$

7 Leifeld L, Germer CT, Bohm S et al. S2k guidelines diverticular disease/ diverticulitis. Zeitschrift fur Gastroenterologie 2014; 52: 663 - 710

8 Parks TG. Natural history of diverticular disease of the colon. A review of 521 cases. British medical journal 1969; 4: 639-642

9 West AB, Losada M. The pathology of diverticulosis coli. Journal of clinical gastroenterology 2004; 38: S11-S16

10 Commane DM, Arasaradnam RP, Mills $S$ et al. Diet, ageing and genetic factors in the pathogenesis of diverticular disease. World journal of gastroenterology 2009; 15: 2479-2488

11 Delvaux M. Diverticular disease of the colon in Europe: epidemiology, impact on citizen health and prevention. Alimentary pharmacology \& therapeutics 2003; 18: $71-74$

12 Etzioni DA, Mack TM, Beart RW Jr et al. Diverticulitis in the United States: 1998-2005: changing patterns of disease and treatment. Annals of surgery 2009; 249: 210-217

13 Granlund J, Svensson T, Olen $O$ et al. The genetic influence on diverticular disease - a twin study. Alimentary pharmacology \& therapeutics 2012; 35: $1103-1107$

14 Painter NS, Burkitt DP. Diverticular disease of the colon: a deficiency disease of Western civilization. British medical journal 1971; 2: 450-454

15 Lin OS, Soon MS, Wu SS et al. Dietary habits and right-sided colonic diverticulosis. Diseases of the colon and rectum 2000; 43: 1412-1418

16 Manousos ON, Vrachliotis G, Papaevangelou G et al. Relation of diverticulosis of the colon to environmental factors in Greece. The American journal of digestive diseases 1973; 18: 174-176

17 Peery AF, Barrett PR, Park D et al. A high-fiber diet does not protect against asymptomatic diverticulosis. Gastroenterology 2012; 142: $266-272$ e 1

18 Song JH, Kim YS, Lee JH et al. Clinical characteristics of colonic diverticulosis in Korea: a prospective study. The Korean journal of internal medicine 2010; 25: 140-146 
19 Strate $L L$, Liu YL, Syngal S et al. Nut, corn, and popcorn consumption and the incidence of diverticular disease. Journal of the American Medical Association 2008; 300: 907-914

20 Strate LL, Liu YL, Aldoori WH et al. Obesity increases the risks of diverticulitis and diverticular bleeding. Gastroenterology 2009; 136: $115-122$ e 1

21 Hwang SS, Cannom RR, Abbas MA et al. Diverticulitis in transplant patients and patients on chronic corticosteroid therapy: a systematic review. Diseases of the colon and rectum 2010; 53: 1699-1707

22 Poncet G, Heluwaert F, Voirin D et al. Natural history of acute colonic diverticular bleeding: a prospective study in 133 consecutive patients. Alimentary pharmacology \& therapeutics 2010; 32: 466 - 471

23 Kaser SA, Fankhauser G, Glauser PM et al. Diagnostic value of inflammation markers in predicting perforation in acute sigmoid diverticulitis. World journal of surgery 2010; 34: 2717-2722

24 Toorenvliet BR, Bakker RF, Breslau PJ et al. Colonic diverticulitis: a prospective analysis of diagnostic accuracy and clinical decision-making. Colorectal disease: the official journal of the Association of Coloproctology of Great Britain and Ireland 2010; 12: 179-186

25 Laurell H, Hansson LE, Gunnarsson U. Acute diverticulitis - clinical presentation and differential diagnostics. Colorectal disease 2007; 9: 496-501

26 Schwerk WB, Schwarz S, Rothmund M. Sonography in acute colonic diverticulitis. A prospective study. Diseases of the colon and rectum 1992; 35: 1077-1084

27 Pradel JA, Adell JF, Taourel P et al. Acute colonic diverticulitis: prospective comparative evaluation with US and CT. Radiology 1997; 205: $503-512$

28 Schreyer AG, Menzel C, Friedrich C et al. Comparison of high-resolution ultrasound and MR-enterography in patients with inflammatory bowel disease. World journal of gastroenterology 2011; 17: 1018 - 1025

29 Lameris W, van Randen A, Bipat $S$ et al. Graded compression ultrasonography and computed tomography in acute colonic diverticulitis: meta-analysis of test accuracy. European radiology 2008; 18: 2498 2511

30 Liljegren G, Chabok A, Wickbom M et al. Acute colonic diverticulitis: a systematic review of diagnostic accuracy. Colorectal disease: the official journal of the Association of Coloproctology of Great Britain and Ireland 2007; 9: 480-488

31 Gielens MP, Mulder IM, van der Harst E et al. Preoperative staging of perforated diverticulitis by computed tomography scanning. Techniques in coloproctology 2012; 16: 363-368
32 Tack D, Bohy P, Perlot I et al. Suspected acute colon diverticulitis: imaging with low-dose unenhanced multi-detector row CT. Radiology 2005; 237: 189-196

33 Heverhagen JT, Sitter H, Zielke A et al. Prospective evaluation of the value of magnetic resonance imaging in suspected acute sigmoid diverticulitis. Diseases of the colon and rectum 2008; 51: 1810-1815

34 Schreyer AG, Furst A, Agha A et al. Magnetic resonance imaging based colonography for diagnosis and assessment of diverticulosis and diverticulitis. International journal of colorectal disease 2004; 19: 474-480

35 Ajaj W, Ruehm SG, Lauenstein T et al. Dark-lumen magnetic resonance colonography in patients with suspected sigmoid diverticulitis: a feasibility study. European radiology 2005; 15: 2316 - 2322

36 Sakhnini E, Lahat A, Melzer E et al. Early colonoscopy in patients with acute diverticulitis: results of a prospective pilot study. Endoscopy 2004; 36: 504-507

37 Chintapalli KN, Chopra S, Ghiatas AA et al. Diverticulitis versus colon cancer: differentiation with helical CT findings. Radiology 1999; 210: 429-435

38 Oistamo E, Hjern F, Blomqvist L et al. Cancer and diverticulitis of the sigmoid colon. Differentiation with computed tomography versus magnetic resonance imaging: preliminary experiences. Acta radiologica 2013; 54: 237-241

39 Bahadursingh AM, Virgo KS, Kaminski DL et al. Spectrum of disease and outcome of complicated diverticular disease. American journal of surgery 2003; 186: 696-701

40 Siewert B, Tye G, Kruskal J et al. Impact of CT-guided drainage in the treatment of diverticular abscesses: size matters. Am J Roentgenol American journal of roentgenology 2006; 186: 680-686

41 Brandt D, Gervaz P, Durmishi $Y$ et al. Percutaneous CT scan-guided drainage vs. antibiotherapy alone for Hinchey II diverticulitis: a casecontrol study. Diseases of the colon and rectum 2006; 49: 1533-1538

42 Zuckerman GR, Prakash C. Acute lower intestinal bleeding. Part II: etiology, therapy, and outcomes. Gastrointestinal endoscopy 1999; 49: $228-238$ 